\title{
28 Research Soure \\ Going Digital: Added Value of Electronic Data Collection in 2018 Afghanistan Health Survey
}

Christina Mergenthaler ( $\nabla$ c.mergenthaler@kit.nl )

Royal Tropical Institute

\section{Rajpal Singh Yadav}

Particip GmbH

\section{Sohrab Safi}

Particip GmbH

\section{Ente Rood}

Royal Tropical Institute

\section{Sandra Alba}

Royal Tropical Institute

\section{Research Article}

Keywords: Digital data collection, tablets, household survey, OpenDataKit, Afghanistan

Posted Date: March 9th, 2021

DOl: https://doi.org/10.21203/rs.3.rs-244356/v1

License: (c) (1) This work is licensed under a Creative Commons Attribution 4.0 International License. Read Full License 


\section{Abstract}

Background: Through a nationally representative household survey in Afghanistan, we conducted an operational study in two relatively secure provinces comparing effectiveness of electronic data collection (EDC) with paper data collection (PDC).

Methods: In Panjshir and Parwan provinces, household survey data were collected using paper questionnaires in 15 clusters, versus OpenDataKit (ODK) software on electronic tablets in 15 clusters. Added value was evaluated from three perspectives: efficient implementation, data quality, and acceptability. Efficiency was measured through financial expenditures and time stamped data. Data quality was measured by examining completeness. Acceptability was studied through focus group discussions with survey staff.

Results: Training, printing, material procurement, wages and transportation costs were $68 \%$ more expensive in electronic clusters compared to paper clusters, due to upfront one-time investment for survey programming. Enumerators spent significantly less time administering surveys in electronic cluster households (248 minutes survey time) compared to paper (289 minutes), for an average savings of 41 minutes per household ( $95 \% \mathrm{Cl}: 25-55)$. EDC offered a savings of 87 days for data management over PDC.

Among 49 tracer variables (meaning responses were required from all respondents), small differences were observed between PDC and EDC. 2.2\% of the cleaned dataset's tracer data points were missing in electronic surveys $(1,216 / 56,073$ data points), compared to $3.2 \%$ in paper surveys $(1,953 / 60,675$ data points). In pre-cleaned datasets, $3.9 \%$ of tracer data points were missing in electronic surveys $(2,151$ / 55,092 data points) compared to $3.2 \%$ in paper surveys $(1,924 / 60,113$ data points).

Enumerators from Panjsher and Parwan preferred EDC over PDC due to time savings, user-friendliness, improved data security, and less conspicuity when traveling; however approximately half of enumerators trained from all 34 provinces reported feeling unsafe due to Taliban presence. Community and household respondent skepticism could be resolved by enumerator reassurance. Enumerators shared that in the future, they prefer collecting data digitally when possible.

Conclusions: EDC offers clear gains in efficiency over PDC for data collection and management time, although costs are relatively comparable even without the programming investment. However, serious field staff concerns around Taliban threats and general insecurity mean that EDC should only be conducted in relatively secure areas.

\section{Background}

As of 2020, data for many complex household surveys, including Demographic and Health Surveys (DHS), are still collected using paper, which demands substantial material resource procurement, organizational planning around paper storage, and transportation and entry into the final electronic 
systems. This has consequences in terms of time, human and financial resources, and seriously tests the capacity of research groups implementing the survey. Researchers in even fragile and unstable settings who are already strapped for time and resources recognize that electronic data collection (EDC) can seriously reduce this strain [1]. Yet there is very limited data comparing the performance of PDC versus $E D C$ in fragile and conflict-affected settings. From the standpoint of public health research we were interested to evaluate the differences in time and cost efficiency of training, procurement and data collection; data quality; and user perceptions of these two methods in insecure settings. The 2018 Afghanistan Health Survey, which aimed to describe key maternal, child and general population health metrics and access issues, provided an opportunity to compare the above aspects of PDC versus EDC. The full report of the survey is available online [2], while the aim of the study presented in this manuscript was to assess the added value of EDC over PDC from three perspectives: efficiency of implementation, data quality, and acceptability.

Use of mobile phones or tablets for digital data collection by researchers and health care workers is increasingly common in low-income countries $[3,4,5,6]$. Investigators of the Digital Engagement and Resilience (DEAR) project of University of Aberdeen described how the uptake of digital technology at the individual and group level is influenced by societal and information technology ecosystem factors [7]. A framework developed to evaluate eHealth solutions addresses the need to cater to different end users and to address environmental (ecosystem) factors which enable use of the technology [8]. Based partially on a number of factors from the DEAR project framework and the existing body of research we developed a theoretical framework to evaluate the use of digital technology in a survey setting, across three concentric environments (Fig. 1). This framework intends to capture the factors which affect the quality, efficiency and acceptability of digital data uptake from the stage of household data survey collection to data analysis and broad-scale dissemination. While a number of online resources provide guidance on the use of tablets to collect and analyze data $[9,10]$, limited studies document issues around acceptability and feasibility in a rigorous way [3].

The 2018 Afghanistan Health Survey was conducted in all 34 provinces of the country to collect nationally representative data on household sociodemographic, maternal and child health indicators. To the best of our knowledge, this is the first nationally representative survey with digital components in Afghanistan. Data collection was done using paper forms throughout the country except for two relatively secure provinces Panjshir and Parwan, where study coordinators aimed to measure the added value of EDC compared to PDC. More secure settings were chosen for this study as the Taliban has been known to capture and interrogate field researchers and health workers at security checkpoints [11], particularly those with valuable equipment such as laptops or tablets [12].

Our objective was to evaluate data efficiencies, user acceptability and feasibility in the 2018 Afghanistan Health Survey across the three environments of digital technology uptake: the respondent - enumerator environment, the data management and analysis environment, and the political and technological environment. The aim of this study is provide evidence for the scale up of digital survey technology in future surveys in Afghanistan and other fragile and conflict affected settings. 


\section{Methods}

For each stage of the framework we developed we determined which indicators should be evaluated to measure this uptake. For the Afghanistan Health Survey we applied a two-stage cluster design with the first stage as the province consisting of 15 clusters per province, and the second stage as cluster with 23 households per cluster. To compare efficiency, data quality and acceptability of PDC versus EDC, we carried out a mixed methods study in two of the 34 provinces: Panjshir and Parwan (Fig. 2). We used both quantitative reanalysis of survey processes and output data as well as qualitative analysis of survey staff focus group discussions.

In both Panjshir and Parwan, household survey data were collected on paper questionnaires in 15 clusters, and using electronic data collection software (in our case OpenDataKit [ODK]) installed on electronic tablets in the remaining 15 clusters. The data management unit (DMU) was responsible for electronic entry of paper-based data, data coding, verification and validation. The DMU was led by a data manager and deputy data manager, data entry supervisor, data officer, 20 data entry operators, and five data coding officers.

\section{Timeliness and Cost Efficiency}

We assessed time taken and expenditures made to collect and process data to determine whether any efficiencies were gained by collecting data electronically. For this purpose we reviewed detailed financial expenditure reports involved in preparation and execution of the household survey in Panjshir and Parwan provinces. We analyzed time stamps for key data collection and data management steps: 1) start and stop time stamps for each of six household survey modules and; 2) start and stop time stamps for data coding, first data entry and second (verification) data entry. Data collection time stamps were available in the study databases as these were automatically collected in the data collection software or manually written in the paper-based household survey questionnaires. Data coding and entry time stamps were manually logged by data management staff for paper-based questionnaires only. We used these to calculate hours spent coding and entering data and subtracted one hour from each day worked to account for breaks and rest. EDC did not require substantial time investments for data coding, entry or verification because they were already collected and stored electronically on the study's server database (ODK Aggregate in our case), so no direct comparison between the time spent coding and verifying paper and digitally collected data could be made. Calculations around human resource time factored in hours worked by the entire DMU team.

\section{Data Completeness and Consistency}

Data quality was measured by examining completeness of data points (i.e. the number of household respondents multiplied by the number of expected responses per respondent) in the pre-cleaned database and the final database of both the PDC and EDC. The pre-cleaned database contains the doubly entered electronic data from the paper-based surveys, and the raw data uploaded to the server from electronic tablets. The final database contains the data which, when collected on paper, were then coded and 
doubly entered, and when electronically collected, were also coded and then received further cleaning (i.e. merging household members and de-duplicating). Due to survey skip logic, household respondents were asked different numbers and combinations of questions. We standardized the measure of completeness across all respondents by studying the questions which everybody was asked. Therefore we identified forty-nine tracer questions which the survey required from each respondent out of a total of $295^{1}$ possible survey questions, regardless of any answers they supplied for any other questions. Per household, we calculated the ratio of complete tracer data points to total possible tracer data points in the pre-cleaned and final paper and electronic datasets, for each survey module. Data cleaning removed some data points from the final datasets of both ODK and paper-based data, for example those data which were collected due to incorrectly applied skip logic, or were clearly illogical.

\section{Acceptability}

We compared acceptability of paper-based and electronically collected data from the perspective of survey field staff; in total there was one team per province consisting of three male and three female enumerators, one supervisor, and for paper clusters only one data editor. We conducted three focus group discussions (FGD): one female and one male group, each with four enumerators and one field editor, and one separate FGD consisting of four field team supervisors (two female and two male). In each FGD, the survey staff present conducted both the paper-based surveys and electronic survey clusters. Focus groups were interviewed in a semi-structured fashion about perceptions of user-friendliness, difficulties in the field, efficiency, skip logic, safety of use, and enumerator-respondent interactions. We later administered a two-question survey about safety and comfort during digital data collection to field staff trainees for two subsequent nationally representative surveys (HMIS verification and BSC), to get the perspectives for insecure areas.

${ }^{1} 295$ questions were possible for household respondents and 26 additional metadata points were associated with each listed household member.

\section{Results}

\section{Does electronic data collection result in more efficient survey implementation?}

\section{Timeliness}

For each individual module, enumerators in electronic cluster households spent significantly less time administering the questionnaire, for an average time savings of 7.4 minutes per module per household (95\% Cl: 6.6-8.2 minutes) (Fig. 3). Comparing the time required to administer the entire survey in a household, enumerators also spent significantly less time administering them in electronic cluster households (248 minutes total survey investment time [95\% Cl: 239.1-258.1]) compared to paper 
clusters (289 minutes [95\% Cl: 277.8-299.8]), for an average savings of 40 minutes per household (95\% Cl: 25.4-55.1 minutes).

Paper-based questionnaires contained responses which needed to be coded into categories to allow for data analysis. Parwan DMU staff spent an average of 5 minutes and 54 seconds per household to code responses, while in Panjshir DMU staff spent an average of 8 minutes and 33 seconds to code responses per household. The first entry from paper into the electronic database of one Parwan household of data required an average of 18 minutes and 40 seconds; the verification entry required an average of 14 minutes and 55 seconds. Panjshir's first entry household average time was 13 minutes and 40 seconds, while the verification data entry required an average of 13 minutes and 16 seconds. Additional quality control performed by two lead DMU staff required approximately 9 eight-hour working days, or an average of 18 minutes per household.

Programming electronic surveys for use in ODK software lasted approximately 73.5 eight-hour working days. Data were transferred to the server database using WiFi hotspots installed via tablet sim cards. Coding the electronically collected data required two eight-hour working days, and no verification entry was performed or necessary. Additional data cleaning and quality control performed by two lead DMU staff required about 7 eight-hour working days each. Figure 4 presents the time used for all data management tasks (programming and quality control for ODK; coding, data entry and quality control for paper) on a household level. Average time spent for all data management tasks was approximately 74 minutes for paper-based households and 66 minutes for ODK households. Total data management for ODK data took a total of 719 hours (90 person-days), only 131 hours (16.4 person-days) of which were spent for processing (coding and cleaning). PDC data coding, entry, management and cleaning alone consumed a total of 812 hours (101.5 person-days).

\section{Cost Efficiency}

The actual combined expenditures in personnel fees and transportation, data management, training, procurement of equipment, and printing amounted to a per province total of 59,958 USD spent for the combined electronic clusters, and 35,762 USD spent for the combined paper clusters (Table 1). Field team personnel costs (fees and transportation) comprised the majority of these costs at 26,100 USD in both paper and electronic clusters, covering six interviewers, one data editor, and one supervisor for each province. These teams of eight conducted surveys in both the paper and electronic clusters of each province. The largest cost difference resulted from the investment required to electronically program the survey, increasing the cost for EDC clusters by 19,634 USD. Costs to develop the survey questionnaires into a word processor were not factored into this analysis. Training costs for EDC staff exceeded those of the PDC staff by 2,826 USD because they received both PDC and EDC training. This was necessary because EDC staff also performed paper-based surveys in the remaining 32 provinces of Afghanistan, and these skills certainly contributed to the quality and efficiency of the work performed by survey staff in the EDC clusters. Equipment costs of 2,157 USD for ECD cluster tablets and charging devices were not required in the paper clusters, which only had an additional 516 USD of printing costs for the paper forms 
not needed in the electronic clusters. Per household the EDC method cost 91.12 USD, while PDC methods cost 54.43 USD per household.

When initial costs associated with EDC are removed (one-time survey programming and equipment costs), the total cost for EDC would have been 38,346 USD. This however does not account for efficiencies which would be gained over time for data cleaning and management, and includes one replacement tablet, power bank, an internet package, umbrella and other equipment. The per household costs would also be more comparable to the PDC costs at 58.28 USD. 
Table 1

Cost Comparison of Paper and Electronic Data Collection and Management

Line item

Paper

ODK

Clusters

ODK Clusters without upfront programming costs

Field Team Personnel (fees and transportation) costs:

\begin{tabular}{llll} 
Interviewers (six people per province) & $\$ 13,500.00$ & $\$ 13,500.00$ & $\$ 13,500.00$ \\
\hline Editor (one person per province) & $\$ 2,700.00$ & $\$ 2,700.00$ & $\$ 2,700.00$ \\
\hline Supervisor (one person per province) & $\$ 9,900.00$ & $\$ 9,900.00$ & $\$ 9,900.00$ \\
\hline Total: & $\mathbf{\$ 2 6 , 1 0 0 . 0 0}$ & $\mathbf{\$ 2 6 , 1 0 0 . 0 0}$ & $\mathbf{\$ 2 6 , 1 0 0 . 0 0}$
\end{tabular}

Data management costs:

Survey programming costs

Data entry and coding costs

$\$ 0.00 \quad \$ 19,634.40 \quad \$ 0.00$

Data cleaning and management costs

$\$ 1,082.25 \quad \$ 0.00 \quad \$ 0.00$

Total:

$\$ 1,256.40 \quad \$ 2,432.65 \quad \$ 2,432.65$

$\$ 2,338.65 \quad \$ 22,067.05 \quad \$ 2,432.65$

Training costs:

Standard training cost to AHS field staff

Electronic data collection training for

Panjshir and Parwan

Electronic data collection refresher training $\quad \$ 0.00 \quad \$ 1,413.19 \quad \$ 1,413.19$ for Panjshir and Parwan

Total:

$\$ 6,807.33 \quad \$ 9,633.71 \quad \$ 9,633.71$

Equipment costs:

Costs of tablets for electronic data collection

Costs of power banks

$\$ 6,807.33 \quad \$ 6,807.33 \quad \$ 6,807.33$

$\$ 0.00 \quad \$ 1,413.19 \quad \$ 1,413.19$

Costs of internet for data transfer

Costs of umbrellas

$\$ 0.00$

$\$ 1,729.41$

$\$ 144.12$

Costs of other equipment

Total:

$\$ 0.00$

$\$ 247.06$

$\$ 20.59$

$\$ 0.00$

$\$ 40.00$

$\$ 3.33$

$\$ 0.00$

$\$ 17.65$

$\$ 1.47$

$\$ 0.00$

$\$ 123.53$

$\$ 10.29$

$\$ 0.00$

$\$ 2,157.65$

$\$ 179.80$

Printing costs:

Printing costs for paper survey forms

$\$ 516.18$

$\$ 0.00$

$\$ 0.00$ 


\begin{tabular}{|llll|}
\hline Line item & $\begin{array}{l}\text { Paper } \\
\text { Clusters }\end{array}$ & $\begin{array}{l}\text { ODK } \\
\text { Clusters }\end{array}$ & $\begin{array}{l}\text { ODK Clusters without } \\
\text { upfront programming } \\
\text { costs }\end{array}$ \\
\hline Costs of photocopying paper & $\$ 0.00$ & $\$ 0.00$ & $\$ 0.00$ \\
\hline Total: & $\$ 516.18$ & $\$ 0.00$ & $\$ 0.00$ \\
\hline Data collection type total: & $\$ 35,762.16$ & $\$ 59,958.41$ & $\$ 38,346.17$ \\
\hline Data collection type total per household: & $\$ 54.43$ & $\$ 91.12$ & $\$ 58.28$ \\
\hline
\end{tabular}

\section{Does electronic data collection result in improved data quality?}

\section{Data Completeness and Consistency}

Differences in data quality were evaluated from the perspectives of completeness and internal consistency. In each module we calculated the number of missing data points out of all possible required data points for the forty-nine tracer variables only. For the pre-cleaned data (as defined above), the paperbased dataset was missing a slightly higher percentage of tracer data points $(2.0 \%: 1,202$ / 59,424) compared to the electronically collected dataset (1.6\%: 911 / 55,576) (Fig. 5a).

After data cleaning, the electronically collected dataset was still missing slightly fewer tracer variable data points (1.1\%: $626 / 55,466)$ than the paper dataset $(2.0 \%: 1,223 / 59,980)$ (Fig. 5b). In both the precleaned and final datasets paper-based data only modules on women's health and immunization were lacking any tracer data. The electronically collected dataset missed tracer data points in all modules, but primarily in the women's and immunization modules. One question related to children's vaccination cards was unexpectedly routinely skipped, accounting for more than $95 \%$ of the missing data points in the immunization module.

\section{How acceptable did field enumerators and respondents find electronic data collection compared to paper based data collection?}

\section{Clarity and ease of use}

Data collectors were interviewed about clarity and ease of using electronic versus paper forms for data collection. Most enumerators preferred electronic data collection over paper due to substantial time savings, user-friendliness, improved data security, and less conspicuity when traveling. These benefits of 
EDC were cited in all three focus group discussions. Automated skip logic was the most frequently mentioned reason for preferring EDC. This removed the necessity to manually decide which questions need to be asked based on previous responses, as the electronic program did this for data collectors. Many enumerators mentioned that this was a helpful time-saver during data collection.

Enumerators also appreciated the logic constraints of EDC, and that certain fields did not make it possible to enter impossible answers, for example an age which is too high or too low. In terms of practical convenience, some enumerators reported that tablets were preferable to paper when it was raining, as papers would sometimes become wet and difficult to read, while a tablet is easier to protect from rain. Data collectors also enjoyed that tablets were lighter to carry than large piles of paper questionnaires.

Some limitations around EDC were noted. A few data collectors reported that previously entered information appeared to sometimes be erased when they scrolled back in the questionnaire to correct responses. One enumerator noted that when the tablet memory was full, tablets would turn off, and sometimes tablets would just turn off for no apparent reason. Several enumerators mentioned that when they only recognized mistakes after multiple incorrect or irrelevant responses were entered into the tablet, it was burdensome to remove all incorrect data from the electronic form. With paper it is easy to simply disregard some incorrect responses by crossing them out and writing the correct answer on the spot. EDC moves up this type of data cleaning to the field rather than the data management office.

\section{Interactions with household respondents}

Many of the enumerators mentioned that household respondents were skeptical about safety of their data when they would be entered into tablets. Some household respondents were concerned that the tablets may be used to photograph them or record their voices. This was more of a concern among women than among men. However, they also mostly reported that these concerns were easily resolved by enumerator reassurance about electronic data confidentiality. Conversely some household respondents (generally younger) felt more secure about their information being entered into a tablet rather than paper, because they understood that it would be more difficult for people to get a password to open up the questionnaires on tablets, than it would be to read responses on paper. $54 \%$ (80/ 148) of enumerators in a follow-up survey reported that they would not feel safe and comfortable if they had to use tablets for household data collection. Among those who reported not feeling safe, $22 \%(n=18)$ mentioned Taliban concerns, 33\% $(n=26)$ mentioned concerns around insecurity, 19\% $(n=15)$ mentioned that as tablets are known to collect GPS coordinates, this makes them feel vulnerable to retaliation from either Taliban or communities, and $33 \%(n=26)$ reported that household and community members would be hesitant to accept this practice. Among those working in provinces in which there was Taliban occupancy in 2019 (n $=88), 47$ (53\%) anticipated not feeling safe collecting data in households in the future.

\section{Safety concerns}

When asked which method of data collection they would prefer to use for a hypothetical next survey, most enumerators indicated that EDC is preferred when possible. It was perceived as much more 
comfortable than PDC, more convenient, and by some respondents it was perceived to be more safe even in insecure areas. One can more easily conceal tablets whereas it is often more difficult to hide a large stack of paper questionnaires. The focus group discussions revealed that some enumerators felt unsafe

carrying tablets though due to fears that people may steal them. The two-question survey administered to field staff trainees in all 34 provinces provided more insight into security concerns: $48 \%$ (72/ 148) of enumerators reported that they did not feel safe and comfortable when they used tablets for data collection in a recent health facility survey. Among the 72 respondents who reported not feeling safe, $79 \%$ $(n=57)$ say they are concerned about repercussions from Taliban who view use of smart devices with suspicion due to their capacity to capture GPS coordinates, and subsequent future retaliation from either Taliban or communities. $46 \%(n=33)$ mentioned concerns around general insecurity or theft. Among those working in provinces with Taliban occupancy $(n=88), 38(43 \%)$ reported feeling unsafe using tablets to collect data in health facilities. Enumerators who reported feeling unsafe using tablets in a health facility were 4.5 times more likely (95\% Cl: $2.9-7.2)$ to report anticipate feeling unsafe doing so in a household setting. A few enumerators also mentioned that collecting data electronically in the future depends on the skills of the survey staff. One enumerator mentioned that the only advantage of using paper in the future is that communities more quickly/ easily recognize and accept PDC.

\section{Discussion}

This study offers a systematic assessment of widely accepted indicators of data quality and efficiency within the frame of digital engagement in a conflict-affected setting. Well-maintained original and final databases, and rigorous record-keeping around costs incurred and hours logged were key to the success in comparing quality, efficiency and feasibility of EDC versus PDC in this survey setting. This study suggests that even in fragile and conflict-affected settings, EDC can lead to improved quality and efficiency of data collection and management over PDC, although initial programming investments EDC are costly. Even without upfront programming costs, EDC was 2,584 USD more expensive than PDC, although these could likely be mitigated due to efficiencies gained from increased experience with the data collection software and by consolidating trainings. For repeat surveys in secure settings, electronic programming is preferable as it brings the data management unit more efficiently to the desired end state of electronic storage, even if it is slightly more expensive.

In our study EDC led to an average time savings of 40 minutes per household compared to paper, and when comparing data management and cleaning time alone, EDC saved 87 person-days overall (14.5 compared to 101.5). A comparison of EDC and PDC in households in Burkina Faso showed similar results for saved time in households [13]. Both pre-cleaned and final electronic collected datasets were slightly $(<1 \%)$ more complete than their paper counterparts, which is comparable to findings from similar comparisons in a Kenyan study with $1 \%$ and $0.1 \%$ missing data in paper and electronic datasets respectively [14]. The Burkina Faso study found negligible differences in completeness but duplicates only in paper datasets [13]. A comparison of PDC with tablet-based EDC in northwest Ethiopia also found data completeness to be superior in the electronically collected data [15]. The same study showed that enumerators favored EDC to PDC for similar reasons to those of our study: improved efficiency of data 
collection, improved quality due to automatic skip patterns and data quality checks, faster data transfer and tablets are more convenient to transport.

However, EDC costs exceeded PDC by $68 \%$, but this was almost entirely due to the upfront electronic programming costs of 19,634 USD. These EDC costs far exceeded what other studies have shown. A Kenyan study assessing costs of PDC and EDC found that establishment of a smartphone data collection system was $9.4 \%$ more expensive than PDC, yet over the subsequent two years, EDC was $7 \%$ less expensive to operate compared to PDC [14]. The same Kenyan study also reported that enumerators appreciated the opportunity to improve their IT skills through EDC [15]. Despite the similarity in costs, in the long run, investment in EDC may be a worthwhile investment in relatively secure areas to save data collection time in the field, and data management time in the office. Over time, trainings may become shorter as trainees become increasingly familiar with digital technology, and for repeat surveys, programming time should remain very low. Time is also a valuable resource.

Interviews on acceptability highlighted a number of relevant concerns around the use of digital data in fragile settings: threats to the person(s) carrying the tablet and respondent hesitations were discussed in equal measure. Similar concerns were raised by enumerators of the Ethiopian study [15], and by researchers in a South African study [16]. These issues raise the need to mitigate security risks for field staff using electronic devices in fragile settings and to pre-sensitize communities about safe storage of electronic data in these settings. Local leaders are often engaged prior to household surveys for a similar purpose, yet sharing in advance the news that field staff will pass through an area with valuable electronic devices may further expose them to risk [16].

The efficiencies and user acceptance described above add evidence to the argument for further expansion of digital technology in survey settings, also those affected by insecurity. But total replacement of digital technology over paper is unlikely to occur quickly according to Adner and Kapoor's 'War Between Ecosystems' paradigm, in which the two systems enjoy a 'robust coexistence' dynamic [17]. This particular ecosystem enables both the piloting of digital systems where it is safe to do so, and the use of the 'old technology' of paper. Survey planners are well acquainted with the process of developing paper-based questionnaires and electronically entering paper data, which have been extensively documented in detailed methodological open-source tools and guidelines. Current trends in the technological and political environment suggest that Afghanistan will remain in quadrant two, meaning that digital technology is unlikely to replace paper in the coming years. Challenges abound: digital data collection issues like accessing stable internet connectivity, programming forms, and resolving technological errors usually require specialized knowledge and training. In addition, insecurity also undoubtedly slows down progress towards the next ecosystem evolution.

A number of study limitations may affect the results presented here. First of all, one systemic error affected the calculation of a question in the immunization module. An incorrect skip pattern in the paper questionnaire and subsequent incorrectly programmed skip logic in the electronic version meant that about $40 \%$ of responses were missed for this question. A similar mistake led to high missingness for a 
family planning question in the women's module. Second, time stamps for coding and data entry for paper data were not logged using a precise and consistent approach across all DMU staff, so logged hours could have included non-coding or data entry tasks.

Furthermore, future studies would benefit from including four additional indicators: internal consistency of the final dataset, number of duplicate records, timeliness of data availability, and time for data coding and second-step (verification) entry of electronically collected data (Table 2). Internal consistency was not monitored using a systematic approach; however, we programmed skip logic and field data type constraints into the electronic questionnaire to eliminate user error. The comparison of data completeness and anecdotal evidence suggest that EDC performed better than paper in this regard. For example, six households in paper clusters which did not report having any children under the age of five also contained data on under five illness, while none of the electronic cluster households showed such an inconsistency. 11 respondents from paper clusters responded that a child was born dead, but later responded that the same child is still alive, while no similar irregularities were observed in the electronic cluster data. The same pattern was found in the Ethiopian study, in which invalid entries or errors were more found in nearly half of the paper-based surveys compared to one-third of the electronically collected data [15].

Table 2. Operationalization Table for Evaluation of Digital Data Collection, Management and Analysis.

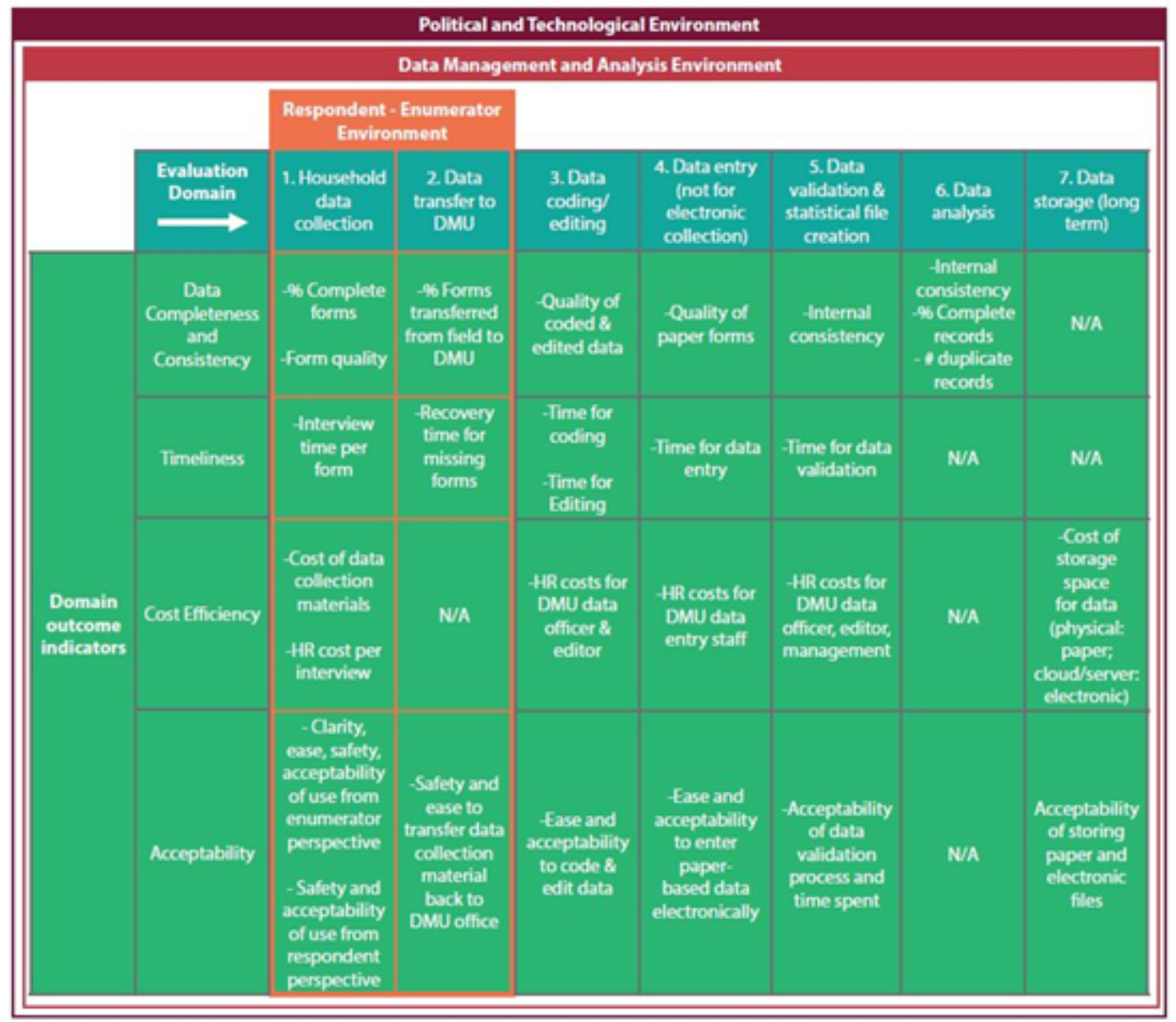




\section{Limitations}

The level of effort required to prepare EDC for coding and verification deserves to be underscored. EDC still required a substantial time investment so that survey modules could be merged for analysis. Due to user error during household data collection, incorrect line numbers were sometimes entered into some modules of the household interviews, resulting in errors during merging, and software-generated record duplication. Although these issues could eventually be resolved in two forty-hour work weeks, it was occasionally necessary to seek information from the field regarding specific households and individuals to merge survey modules correctly. Even in EDC, human error in data entry will lead to logical impossibilities during merging, which different software packages will handle differently [3]. Understanding how one's preferred software will handle many-to-one or one-to-many merging errors is essential before using it to start managing survey data.

\section{Conclusions}

The added value of EDC over PDC was primarily in time efficiencies gained during data collection and processing, although only conducted in relatively secure areas. EDC also resulted in improved acceptability while preserving data quality, despite higher costs. Overall our data provide evidence to support the scale up of digital survey technology in future surveys in Afghanistan and other fragile and conflict affected settings. Hybrid approaches such as ours (in which both paper and digital data collection are deployed) may be appropriate in settings where security varies across space and time.

\section{List Of Abbreviations}

EDC Electronic data collection

PDC Paper data collection

ODK OpenDataKit

DHS Demographic and Health Survey

AHS Afghanistan Health Survey

DMU Data management unit

FGD Focus group discussion

HMIS Health management information system

BSC Balanced Score Card

$\mathrm{Cl}$ Confidence interval 
USD United States Dollars

GPS Global Positioning System

\section{Declarations}

\section{Ethics approval and consent to participate}

For qualitative interviews amongst field staff who were involved in the Afghanistan Health Survey (AHS) 2018, all participants were above the age of 18 and provided oral informed consent. No ethical approval was required for these qualitative interviews because this operational research involved only internal field staff interviews in their professional capacity. We did not ask for any personal or sensitive information from any respondents.

However for the AHS itself, study staff obtained oral informed consent for individuals who were not literate and informed consent from a parent and/or legal guardian of individuals under the age of 18 who were involved in the Afghanistan Health Survey. All methods were carried out in accordance with relevant local and international guidelines and regulations. The AHS did receive ethical approval from Afghanistan's Ministry of Public Health Institutional Review Board and KIT Royal Tropical Institute's Research Ethics Committee.

\section{Consent for publication}

Not applicable

\section{Availability of data and materials}

The datasets generated and/or analysed during the current study are available in the https://zenodo.org repository, [https://zenodo.org/record/4279123\#.X7UBc8hKiUk]. The Afghanistan Health Survey full report can be found available at https://www.kit.nl/wp-content/uploads/2019/07/AHS-2018-reportFINAL-15-4-2019.pdf.

\section{Competing interests}

The authors declare that they have no competing interests

\section{Funding}

All funding was provided by The World Bank Group, whose role was limited to the design of the AHS, but not the design, analysis, or writing of the operational study manuscript.

\section{Acknowledgements}

We acknowledge the valuable contributions of the following individuals in supporting both the AHS and this operational research in particular: Elisabeth Kleipool, Syed Arab, Gilbert Burnham, Khisraw Amani and 
Egbert Sondorp.

\section{References}

1. Dette, R. (2018). Do No Digital Harm: Mitigating Technology Risks in Humanitarian Contexts. In Technologies for Development. https://doi.org/10.1007/978-3-319-91068-0_2

2. Reproductive, Maternal, Newborn, Child and Adolescent Health Directorate (RMNCAH). Ministry of Public Health, I. R. of A. (2018). Afghanistan Health Survey 2018. https://rmncahmoph.gov.af/blog/2019/03/02/ahs-2018/

3. Dickinson, F. M., McCauley, M., Madaj, B., \& Van Den Broek, N. (2019). Using electronic tablets for data collection for healthcare service and maternal health assessments in low resource settings: Lessons learnt. BMC Health Services Research, 19(1), 1-7. https://doi.org/10.1186/s12913-0194161-7.

4. Agarwal, S., Perry, H. B., Long, L. A., \& Labrique, A. B. (2015). Evidence on feasibility and effective use of mHealth strategies by frontline health workers in developing countries: Systematic review. Tropical Medicine and International Health, 20(8), 1003-1014. https://doi.org/10.1111/tmi.12525.

5. King, C., Hall, J., Banda, M., Beard, J., Bird, J., Kazembe, P., \& Fottrell, E. (2014). Electronic data capture in a rural African setting: Evaluating experiences with different systems in Malawi. Global Health Action, 7(1), 1-6. https://doi.org/10.3402/gha.v7.25878.

6. Byass, P., Hounton, S., Ouédraogo, M., Somé, H., Diallo, I., Fottrell, E., Emmelin, A., \& Meda, N. (2008). Direct data capture using hand-held computers in rural Burkina Faso: Experiences, benefits and lessons learnt. Tropical Medicine and International Health, 13(SUPPL. 1), 25-30. https://doi.org/10.1111/j.1365-3156.2008.02084.x.

7. Roberts, E., Farrington, J., \& Skerratt, S. (2015). Evaluating New Digital Technologies Through a Framework of Resilience. Scottish Geographical Journal, 131(3-4), 253-264. https://doi.org/10.1080/14702541.2015.1068947.

8. Dansky, K., Thompson, D., \& Sanner, T. (2006). A framework for evaluating eHealth research. Evaluation and Program Planning, 29(4), 397-404. https://doi.org/10.1016/j.evalprogplan.2006.08.009.

9. Shukla, U., \& Sen, S. (2014). Going Digital: Use of Technology in Monitoring and Evaluation. Abdul Latif Jameel Poverty Action Lab. Second Development Marketplace 2013 Convening Workshop. http://pubdocs.worldbank.org/en/653351463410928020/Core-05-Going-Digital-Use-of-Technologyin-Monitoring-and-Evaluation-Urmy-Shukla-Sree-Sen.pdf.

10. Tomkys, E., \& Sombardini, S. (2015). Going digital: Using digital technology to conduct Oxfam's Effectiveness reviews. OXFAM Case Study. https://oxfamilibrary.openrepository.com/bitstream/handle/10546/578816/cs-going-digitaleffectiveness-reviews-290915-en.pdf;jsessionid=8EF5AF86855745CC90157A63CD129CFD? sequence $=4$. 
11. Faiez, R. (2020). Afghan officials: Taliban attacks on checkpoints kill 29. AP News. https://apnews.com/273a7ea4166e47f8f7a6824e584013d9.

12. Khan, O. F. (2016). Taliban punish people for using smartphones. The Times of India. https://timesofindia.indiatimes.com/world/pakistan/Taliban-punish-people-for-usingsmartphones/articleshow/55809022.cms.

13. Di Pasquale, A. (2018). Improving quality, timeliness and efficacy of data collection and management in population-based surveillance of vital events. [University of Basel]. https://edoc.unibas.ch/64822/1/A_DiPasquale_Thesis.pdf.

14. Njuguna, H. N., Caselton, D. L., Arunga, G. O., Emukule, G. O., Kinyanjui, D. K., Kalani, R. M., Kinkade, C., Muthoka, P. M., Katz, M. A., \& Mott, J. A. (2014). A comparison of smartphones to paper-based questionnaires for routine influenza sentinel surveillance, Kenya, 2011-2012. BMC Medical Informatics and Decision Making, 14(1). https://doi.org/10.1186/s12911-014-0107-5.

15. Zeleke, A. A., Worku, A. G., Demissie, A., Otto-Sobotka, F., Wilken, M., Lipprandt, M., Tilahun, B., \& Röhrig, R. (2019). Evaluation of electronic and paper-pen data capturing tools for data quality in a public health survey in a health and demographic surveillance site, Ethiopia: Randomized controlled crossover health care information technology evaluation. Journal of Medical Internet Research, 21(2). https://doi.org/10.2196/10995.

16. Tomlinson, M., Solomon, W., Singh, Y., Doherty, T., Chopra, M., ljumba, P., Tsai, A. C., \& Jackson, D. (2009). The use of mobile phones as a data collection tool: A report from a household survey in South Africa. BMC Medical Informatics and Decision Making, 9(1), 1-8. https://doi.org/10.1186/1472-6947-9-51.

17. Adner, R., \& Kapoor, R. (2016). Right tech, wrong time. Harvard Business Review, 2016(November), 19. https://doi.org/10.1109/emr.2017.8233298.

\section{Figures}




\section{Political and Technological Environment}

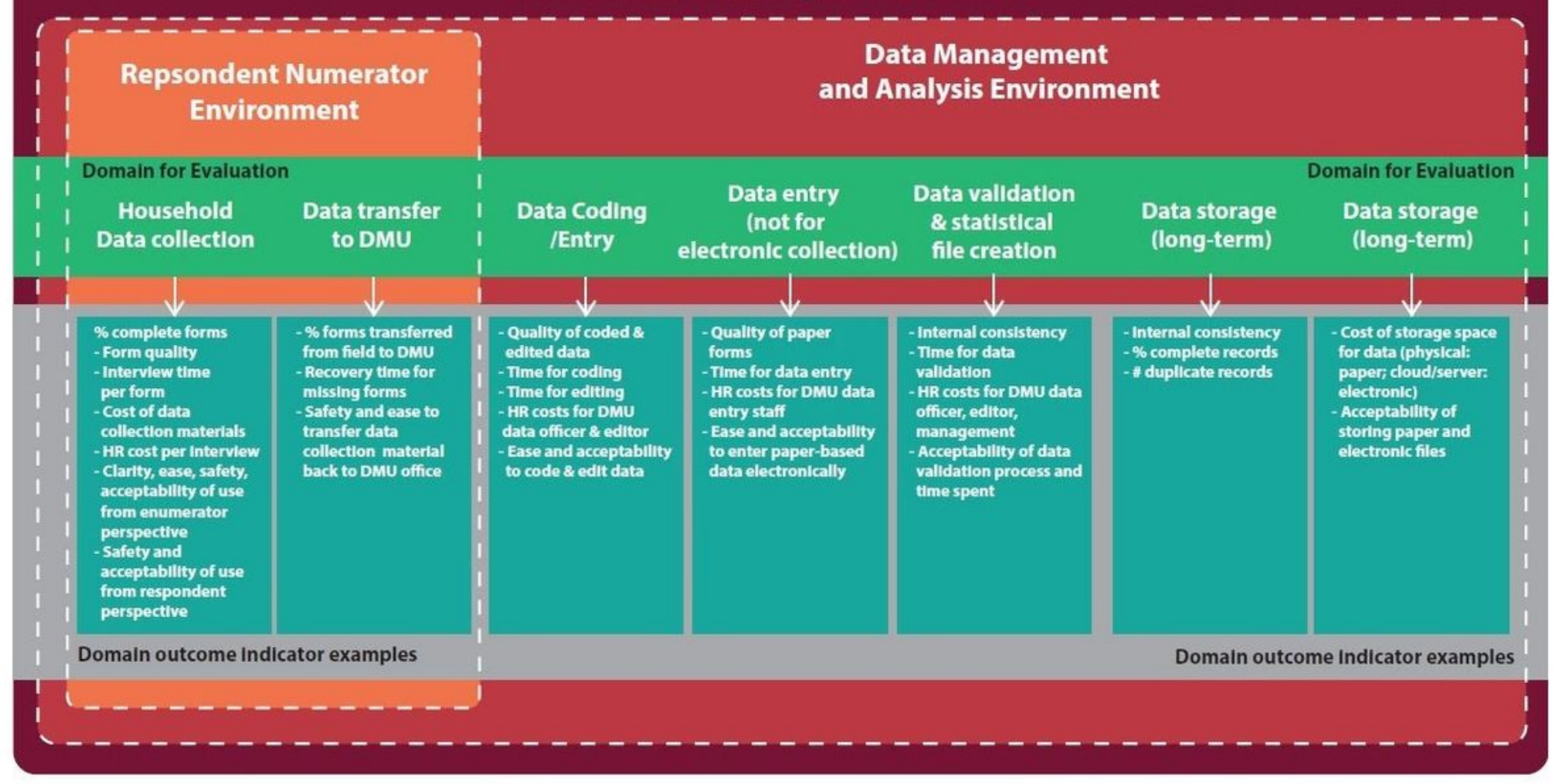

Digital Landscape Factors

- Digital literacy and skills (enumerators)

- Flexibility and agility (enumerators)

- Attitudes to technology (community, respondents,

enumerators)

- Usability/user friendliness

- Trust about confidentiality of data (respondents)

- Resources for materials \& supporting

infrastructure (power banks, tablete)
Digital Landscape Factors

Ability to establish internet connectivity

- Digital competencies of data

management unit

- Leadership of data management unit

- Resources for supporting infrastructure
Digltal Landscape Factors

- Internet Connectivity

- Attitudes to technology

- Leadership

- Regulatory framework and enforcement

for data sharing and enforcement

- Resources for supporting infrastructure

\section{Figure 1}

Conceptual Framework to Evaluate Digital Technology Uptake in Survey Settings 


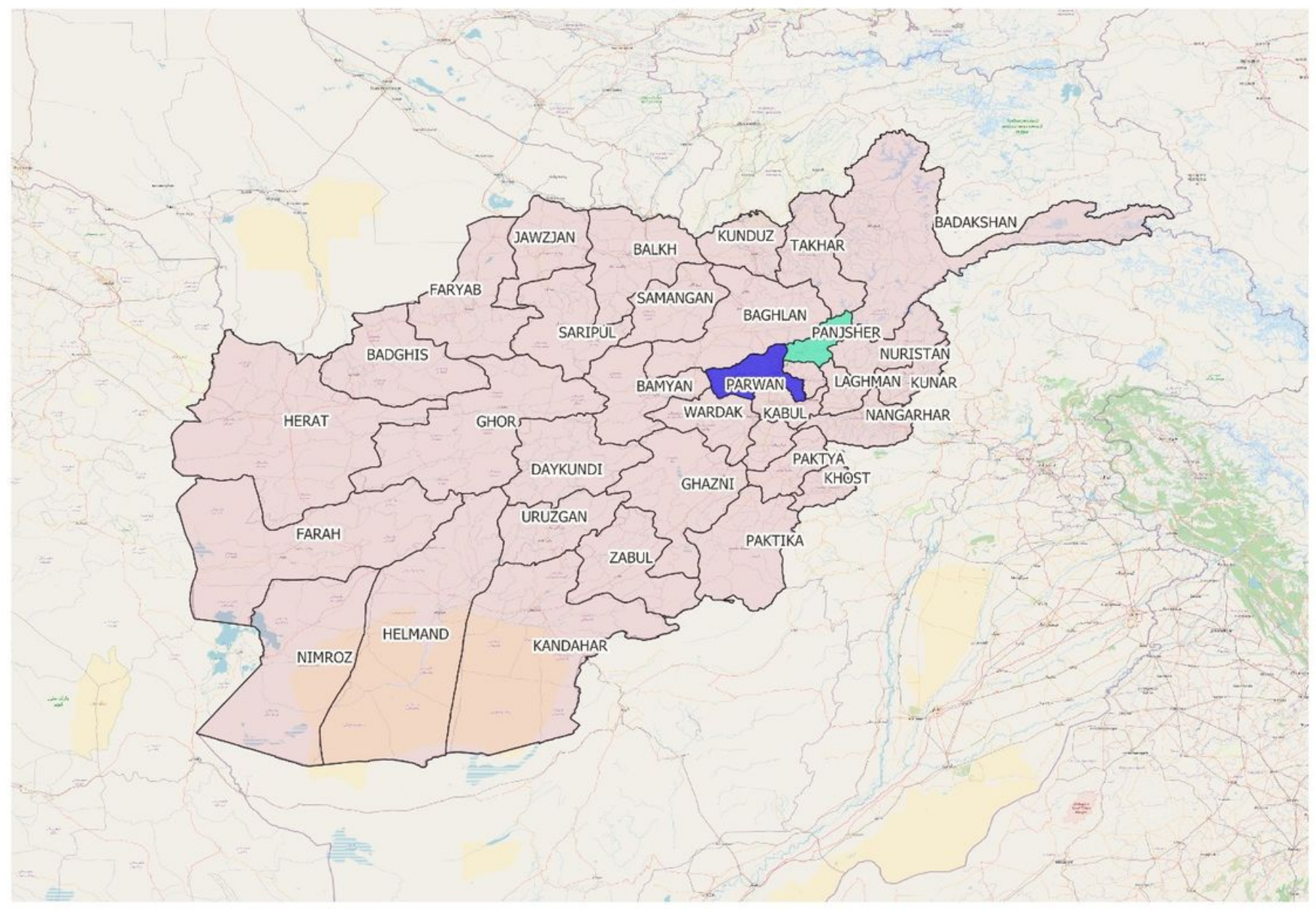

\section{Figure 2}

Location of Panjsher and Parwan ODK Study Sites in 2018 Afghanistan Household Survey Note: The designations employed and the presentation of the material on this map do not imply the expression of any opinion whatsoever on the part of Research Square concerning the legal status of any country, territory, city or area or of its authorities, or concerning the delimitation of its frontiers or boundaries. This map has been provided by the authors. 


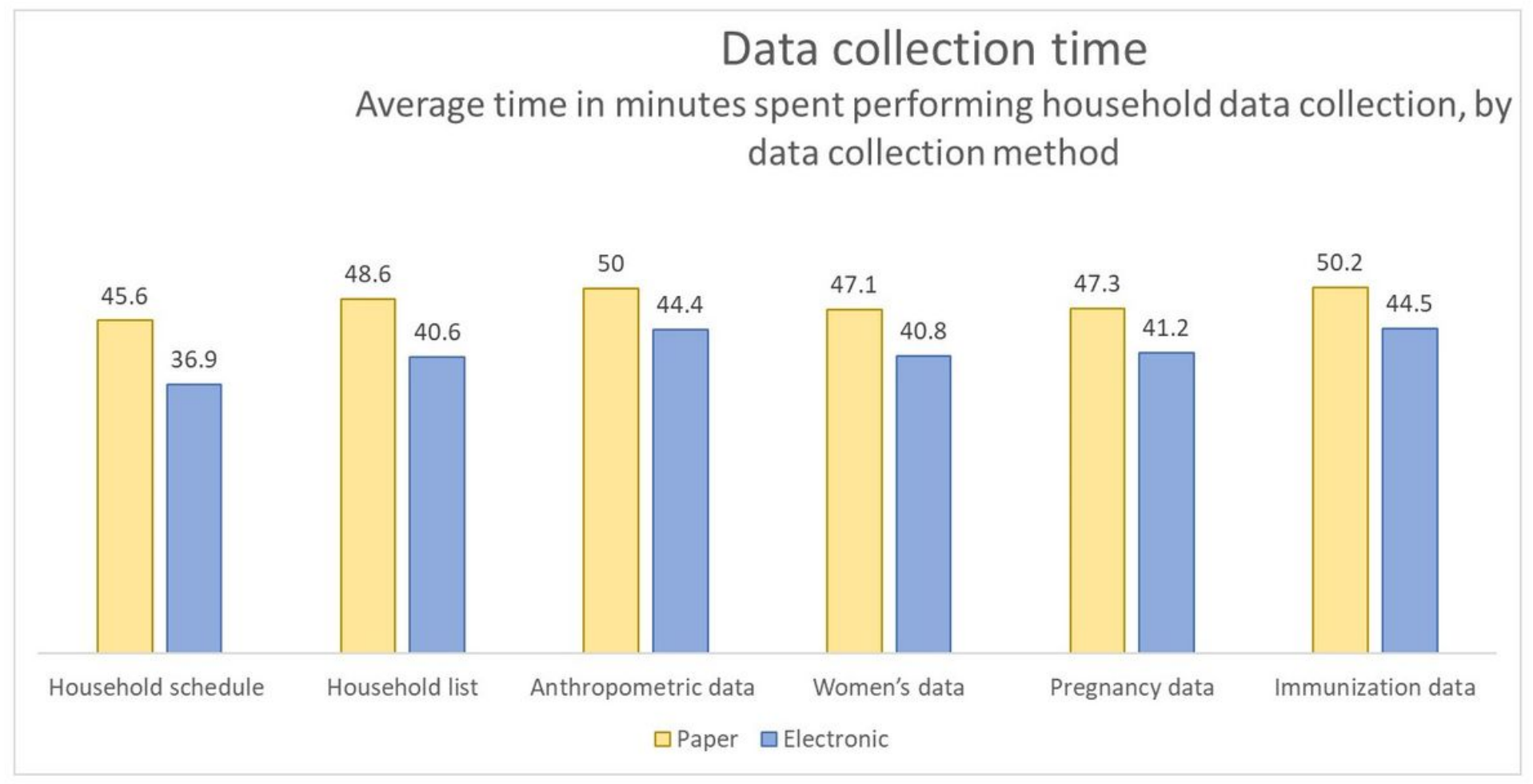

\section{Figure 3}

Average time in minutes spent performing household data collection: PDC vs. EDC.

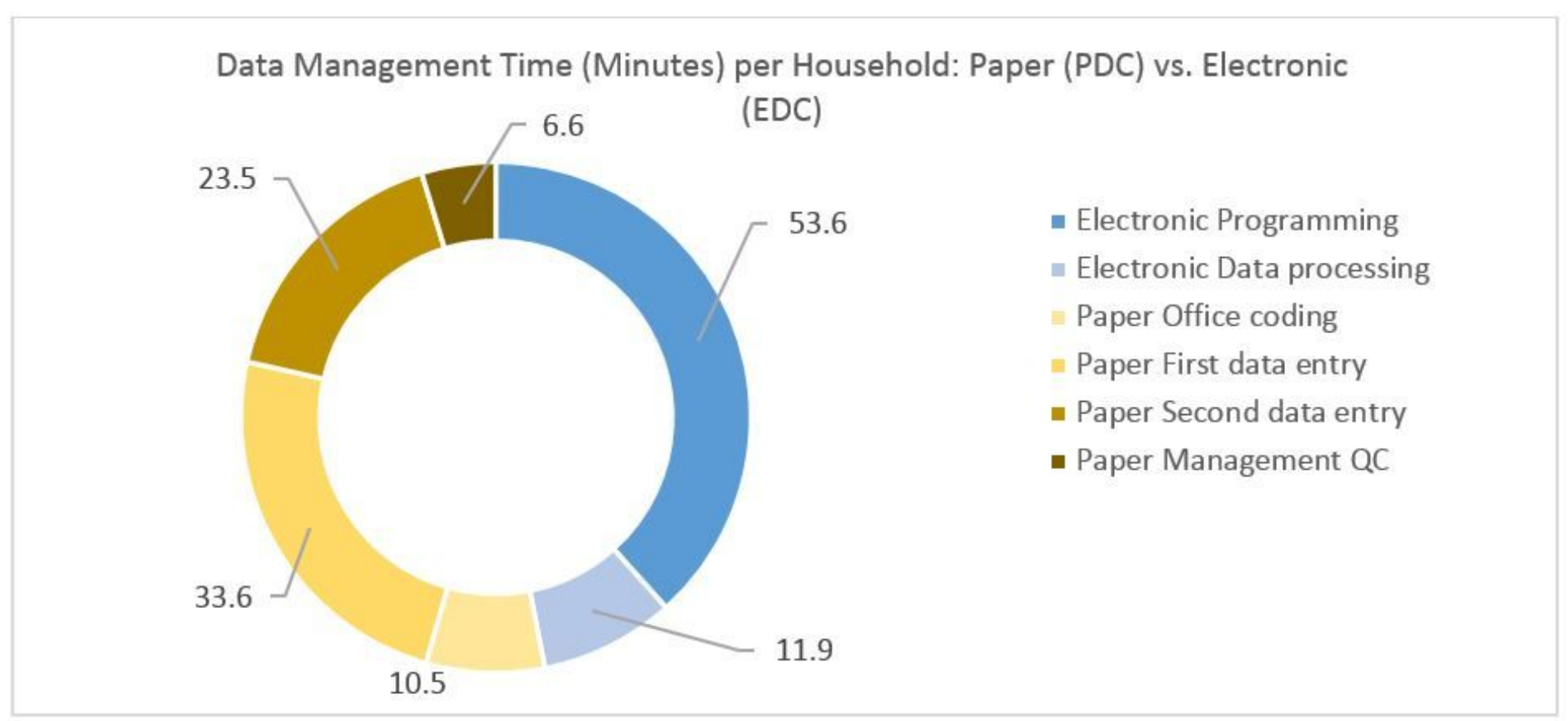

\section{Figure 4}

Time performing data management tasks per household: PDC vs. EDC. 
Figure 5a.

Proportion of missing responses on tracer questions, per module: Pre-cleaned datset

Immunization

Pregnancy

Women

Anthro

Hhlist

Hhdata

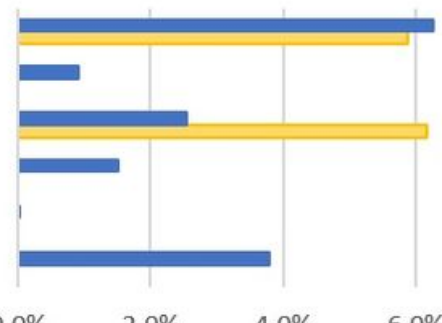

$0.0 \%$

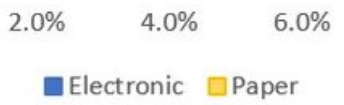

Figure 5b.

Proportion of missing responses on tracer questions, per module: Final dataset

Immunization

Pregnancy

Women

Anthro

Hhlist

Hhdata
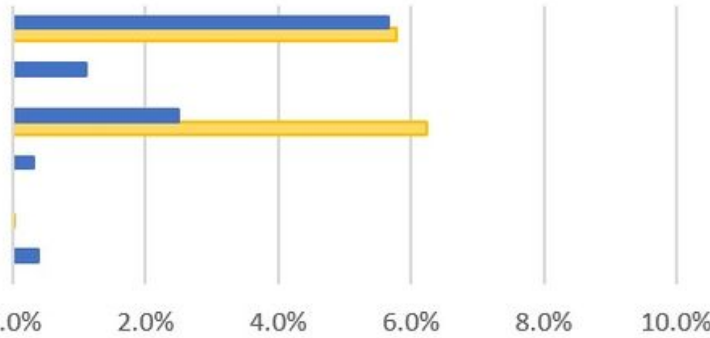

Electronic $\square$ Paper

Figure 5

5a. Pre-cleaned Dataset Completeness for PCD and ECD 5b. Final Dataset Completeness for PCD and ECD 